\title{
Decreased Fast Ripples in the Hippocampus of Rats with Spontaneous Recurrent Seizures Treated with Carbenoxolone and Quinine
}

\author{
Consuelo Ventura-Mejía and Laura Medina-Ceja \\ Laboratory of Neurophysiology and Neurochemistry, Department of Cellular and Molecular Biology, Centro Universitario de Ciencias \\ Biológicas y Agropecuarias (CUCBA), University of Guadalajara, Camino Ing. R. Padilla Sánchez 2100, Las Agujas, Nextipac, Zapopan, \\ JAL, CP 45110, Mexico \\ Correspondence should be addressed to Laura Medina-Ceja; lauramedcej@gmail.com
}

Received 11 June 2014; Accepted 14 August 2014; Published 3 September 2014

Academic Editor: Antonio W. D. Gavilanes

Copyright (C) 2014 C. Ventura-Mejía and L. Medina-Ceja. This is an open access article distributed under the Creative Commons Attribution License, which permits unrestricted use, distribution, and reproduction in any medium, provided the original work is properly cited.

\begin{abstract}
Background. In models of temporal lobe epilepsy and in patients with this pathology, high frequency oscillations called fast ripples (FRs, 250-600 Hz) can be observed. FRs are considered potential biomarkers for epilepsy and, in the light of many in vitro and in silico studies, we thought that electrical synapses mediated by gap junctions might possibly modulate FRs in vivo. Methods. Animals with spontaneous recurrent seizures induced by pilocarpine administration were implanted with movable microelectrodes in the right anterior and posterior hippocampus to evaluate the effects of gap junction blockers administered in the entorhinal cortex. The effects of carbenoxolone (50 nmoles) and quinine (35 pmoles) on the mean number of spontaneous FR events (occurrence of FRs), as well as on the mean number of oscillation cycles per FR event and their frequency, were assessed using a specific algorithm to analyze FRs in intracranial EEG recordings. Results. We found that these gap junction blockers decreased the mean number of FRs and the mean number of oscillation cycles per FR event in the hippocampus, both during and at different times after carbenoxolone and quinine administration. Conclusion. These data suggest that FRs may be modulated by gap junctions, although additional experiments in vivo will be necessary to determine the precise role of gap junctions in this pathological activity associated with epileptogenesis.
\end{abstract}

\section{Introduction}

Epilepsy is a neuronal disorder that is characterized by the abnormal, continued discharge and hypersynchronous activity of neurons [1]. The existing models of epilepsy provide good tools to study the basic mechanisms by which seizures are generated, and the model of temporal lobe epilepsy (TLE) induced by pilocarpine or kainic acid (KA) simulates most of the characteristics of this pathology [24]. In this TLE model, high frequency oscillations known as fast ripples (FRs, 250-600 Hz) have been observed, as in tetrodotoxin and tetanus toxin models of epilepsy [5-7], in computational models in silico $[8,9]$ and in patients with TLE $[2,10,11]$.
FRs can be evoked by electrical stimulation of the same brain areas where they occur spontaneously and they reflect bursts of population spikes from synchronously firing principal cells in relatively small areas of the hippocampus (HIP, $1 \mathrm{~mm}^{3}$ ) [12-14]. According to electrophysiological studies in animals with spontaneous recurrent seizures, these are areas that contain groups of pathologically interconnected neurons $[2,15,16]$. However, FRs have only been recorded in the HIP and entorhinal cortex (EC) ipsilateral to the lesion during slow wave sleep and immobility, albeit for periods ranging from days to months $[2,17]$. In addition, various studies have confirmed that the onset of focal seizures coincides with fast ripple (FR) activity $[10,18,19]$. Accordingly, FRs are considered to be potential biomarkers for epilepsy as they can be 
recorded both before seizure onset and during seizure activity $[3,16]$.

Electrical synapses enable electrical activity to be synchronized between neurons and they permit the spread of depolarization (excitation) or hyperpolarization (inhibition) across a particular neural network [20]. Several studies have highlighted the involvement of electric coupling in epileptiform activity, both in in vitro and in vivo models [19, 20]. Moreover, the loss of electrical coupling provoked by gap junction blockers (e.g., carbenoxolone [CBX], a nonspecific blocker of connexins, or quinine, a dose-dependent blocker of connexin 36) [21,22] or through a deficiency in connexins, the structural proteins that form gap junctions, produces antiepileptic effects [23-30]. Indeed, recent in silico studies [31] supported the participation of gap junctions localized in axons in the generation and persistence of gamma and FR activity.

Based on this information, we have considered the possible involvement of electrical synapses mediated by gap junctions in the modulation of FRs in animals experiencing spontaneous recurrent seizures. For this propose, we evaluated the effects of CBX and quinine on the mean number of spontaneous FR events (FR occurrence), as well as the mean number of oscillation cycles per FR event and frequency.

\section{Methods}

2.1. Model of Temporal Lobe Epilepsy Induced by Intracerebroventricular Pilocarpine Administration. Male Wistar rats (190-200 g) were housed individually in cages in a temperature-controlled room $\left(22 \pm 2^{\circ} \mathrm{C}\right)$, on a $12 \mathrm{~h}$ light/dark cycle (lights on from 7:00 a.m. to 7:00 p.m.) and with ad libitum access to food and water. All experimental procedures were designed to minimize animal suffering and the total number of animals used. The protocols used were in accordance with the Rules for Research in Health Matters (Mexican Official Norms NOM-062-ZOO-1999, NOM-033ZOO-1995) and they were approved by the local Animal Care Committee.

To induce acute status epilepticus (SE) [32], rats were anesthetized with isofluorane (Sofloran, PISA, Laboratories, Mexico) in $100 \%$ oxygen and secured in a Stoelting stereotaxic frame with the incisor bar positioned at $-3.3 \mathrm{~mm}$. A hole was drilled in the skull above the right lateral ventricle at the following stereotaxic coordinates relative to bregma: AP $-4.1 \mathrm{~mm}, \mathrm{~L}-5.2 \mathrm{~mm}$, and V $7 \mathrm{~mm}$. A single dose of pilocarpine hydrochloride $(1.2 \mathrm{mg} / \mu \mathrm{L}$, total volume $2 \mu \mathrm{L}$; SigmaAldrich, USA) was injected through a needle connected to an injection pump (flow rate: $1 \mu \mathrm{L} / \mathrm{min}$; Stoelting Co., IL, USA). After recovery, the animals returned to their cages for observation, convulsive behavior was scored according to the Racine scale [33], and animals with a score of $4 / 5$ were considered to exhibit SE. After 90-120 minutes, SE was abolished by administering diazepam (5-10 $\mathrm{mg} / \mathrm{kg}$, i.p.) to increase their survival and, when necessary, two doses of diazepam were administered. The convulsive behavior of all the animals after pilocarpine injection was monitored visually and 15 days after SE induction the rats were video monitored for 24 hours every day, scoring their spontaneous recurrent seizures. Animals exhibiting spontaneous recurrent seizures were selected for microelectrode implantation.

2.2. Surgery. Control rats were anaesthetized as indicated above and they were positioned in a stereotaxic frame such that lambda and the bregma were in the same horizontal plane. In the control group, fixed recording microelectrodes, consisting of pairs of tungsten wires $(60 \mu \mathrm{m}$ in diameter) with a $1.5 \mathrm{~mm}$ vertical tip separation, were implanted in the right anterior (RAH: AP -3.5 mm; ML $2.00 \mathrm{~mm}$; DV $4.0 \mathrm{~mm}$ ) and posterior hippocampus (RPH: AP $-5.0 \mathrm{~mm}$ relative to bregma; ML $5.0 \mathrm{~mm}$; DV $5.5 \mathrm{~mm}$ ). In addition, two stainless steel screws were driven into the bone, one above the bregma and one above the cerebellum, which served as indifferent and ground electrodes, respectively. Finally, a stainless steel guide cannula $(0.5 \mathrm{~mm}$ internal diameter) was implanted through a hole drilled in the skull and positioned in the region of the right EC (AP $-8.00 \mathrm{~mm}$, ML $4.0 \mathrm{~mm}$, DV $5.0 \mathrm{~mm}$ ) in order to insert a needle for the injection of various chemical agents (CBX, quinine, and saline solution). This arrangement of microelectrodes was placed on a pin connector and, along with the guide cannula, fastened to the skull with dental cement. For experimental groups, an arrangement of eight microelectrodes with the same characteristics as those described above was mounted in a mobile device and implanted into the right region of the hippocampus (RAH, AP $-3.5 \mathrm{~mm}$, ML $3.0 \mathrm{~mm}$, DV $2.5 \mathrm{~mm}$; RPH, AP $-5.0 \mathrm{~mm}$, ML $3.0 \mathrm{~mm}$, DV $2.5 \mathrm{~mm}$ ) in order to move this device to different depths of the hippocampus and easily detect FRs in experiments carried out in freely moving rats.

2.3. Drug Administration. To determine the involvement of electrical synapses, we used three control $(n=3$ rats each one) and three experimental groups of animals ( $n=6$ each one) with recurrent spontaneous seizures, the latter receiving CBX (50 mM, final dose 50 nmoles), quinine (35 mM, final dose 35 pmoles), or the vehicle alone $(\mathrm{NaCl}$ 0.9\%; Sigma Chemical Co. St. Louis, MO, USA). These drugs were administered through an injection needle placed into the guide cannula $(0.2 \mu \mathrm{L} / \mathrm{min}$ flow for 5 minutes $)$ and using a microsyringe mounted to a microinjection pump (WPI, Fl. USA).

2.4. Intracranial EEG Recordings and Analysis. Intracranial EEG activity was recorded in freely moving rats. Five 4channel MOSFET small amplifiers were attached to the cable connector to eliminate movement artifacts. Hippocampal electrical activity was recorded on a polygraph with eight amplifiers (Model 7D, Grass Technologies, RI, USA) at a bandwidth of 0.1 to $3 \mathrm{kHz}$ and with a sensitivity of $75 \mu \mathrm{V} / \mathrm{cm}$ per channel. The sampling rate was set at $5 \mathrm{kHz} / \mathrm{channel}$ with 12-bit precision using an iMac A1048 (Apple, USA) and MP150 software system (BIOPAC Systems, CA, USA). The basal electrical activity of each control group was analyzed, and the amplitude and frequency averaged over a $15 \mathrm{~min}$ recording period. In contrast, once recorded, EEG traces from all recordings of experimental animals were converted 
to MATLAB readable files to process and identify the FRs using an algorithm designed specifically for this purpose [34-36]. Accordingly, each of the signals selected was passed through a $100-650 \mathrm{~Hz}$ band-pass filter using the Hamming method with 60 coefficients. To identify FRs, the filtered signal was processed using the root mean square (RMS) in a sliding window of $3 \mathrm{~ms}$ and through its successive values. Values that were over 5 times the standard deviation of the mean value of the filtered signal and that lasted at least $6 \mathrm{~ms}$ were considered possible FR events. A second criterion was also used for FR classification, whereby the changes in the approximate entropy of the signal were analyzed $[36,37]$ and those that were over twice the mean value were considered putative FRs. Signals that fulfilled these two criteria were processed with the fast Fourier transform (FFT) to calculate their frequency in $\mathrm{Hz}$. In addition, the results from the automated FRs detection algorithm were manually inspected. The FRs parameters evaluated were the mean number of spontaneous FR events (occurrence of FRs per $15 \mathrm{~min}$ ) and the mean number of oscillation cycles per FR event, as well as the frequency before, during, and after CBX or quinine administration.

2.5. Histological Evaluation. After each experiment the animals were anesthetized with sodium pentobarbital and perfused transcardially with $100 \mathrm{~mL}$ of normal saline (0.9\%) in $0.12 \mathrm{M}$ buffer $/ \mathrm{CaCl}_{2}$, followed by $300 \mathrm{~mL}$ of $4 \%$ paraformaldehyde in $0.12 \mathrm{M}$ buffer $/ \mathrm{CaCl}_{2}$ ( $\mathrm{pH}$ 7.3). The animal's brain was then removed, and coronal sections (50 $\mu \mathrm{m}$ thick) were obtained and stained with cresyl violet to confirm the correct positioning of the guide cannula and microelectrodes. Only animals that showed a correct implantation of cannula and microelectrodes were included in the present work.

2.6. Statistical Analysis. In the control groups the data are expressed as the mean \pm SEM of the amplitude and frequency of electrical activity recorded in the RAH, LAH, RPH, and LPH (right/left anterior/posterior hippocampus). Significant differences between control groups were obtained by an analysis of variance (ANOVA, one-way) followed by Tukey's test. The data from the experimental groups are expressed as the mean \pm SEM of each parameter evaluated before, during, and after CBX or quinine administration. Significant differences were analyzed by ANOVA followed by Dunnett's post hoc test, with $P$ values $<0.05$ considered significant.

\section{Results}

3.1. Behavior and Analysis of Intracranial EEG Recordings in Control Animals. Animals from the three control groups showed normal behavior before, during, and after drug administration into the EC (CBX 50 nmoles, quinine 35 pmoles, and $\mathrm{NaCl} 0.9 \%$ ). In these animals normal grooming, chewing movements, exploratory behavior, and sleep periods were observed. The analysis of the intracranial EEG recordings from these control rats revealed a low amplitude and frequency of electrical activity (Figure 1) in all the regions analyzed, both before and after drug administration. The only significant difference observed was in the frequency parameter in the 150-165 min period analyzed between animals from the control groups treated with $\mathrm{NaCl}$ and quinine $(1.16 \pm$ $0.09 \mathrm{~Hz}$ versus $1.55 \pm 0.12 \mathrm{~Hz}$, resp., Figure 1).

\subsection{Effect of Carbenoxolone on the Fast Ripples Observed in} Animals with Spontaneous Seizures. In terms of their behavior, the rats that received CBX showed vibrissae movements, and while they remained in a state of sleep for most of the analysis, spontaneous seizures were observed in some animals at level 5 of the Racine scale during the experiments.

When the intracranial EEG recordings from experimental animals $(n=6)$ with spontaneous and recurrent seizures were analyzed, spontaneous FRs were evident in all the regions registered (Figure 2(a)). The administration of CBX (50 nmoles) produced a significant decrease in the mean number of FRs in the RAH and RPH compared to those that received $\mathrm{NaCl}$ (Figures 3 and 4), both during and at different times after CBX administration (RAH: during $4.8 \pm 1.4$ versus $0.8 \pm 0.2$; after $30-45 \mathrm{~min}, 4.2 \pm 1.4$ versus $0.6 \pm 0.1$; after $150-$ $165 \mathrm{~min} 4.6 \pm 1.7$ versus $0.6 \pm 0.2$. RPH: during, $5.0 \pm 1.5$ versus $0.4 \pm 0.1$; after $30-45 \mathrm{~min}, 6.0 \pm 1.6$ versus $0.5 \pm 0.1$; after $150-$ $165 \mathrm{~min}, 5.8 \pm 1.7$ versus $0.7 \pm 0.2$ ). By contrast, the mean number of oscillation cycles per FR event decreased in the $\mathrm{RPH}$ during and at different times after CBX administration (during $6.3 \pm 1.4$ versus $1.4 \pm 0.5$; after $30-45$ min $6.8 \pm 0.3$ versus $2.9 \pm 0.8$; after $150-165 \min 6.48 \pm 0.2$ versus $2.9 \pm 0.8)$, yet only during CBX administration in the RAH $(5.69 \pm 0.9$ versus $2.5 \pm 0.7$ ). The mean frequency of FRs also decreased in the $\mathrm{RPH}$ compared with the rats that received $\mathrm{NaCl}$ alone, both during and at different times after CBX administration (during 342.1 \pm 77.3 versus 93 \pm 39 ; after $30-45 \min 414.6 \pm 14.5$ versus $175 \pm 51$; after $150-165 \mathrm{~min} 386.6 \pm 10.5$ versus $169 \pm 49$ ), yet only during CBX administration in the RAH (317.2 \pm 51 versus $115 \pm 41 \mathrm{~Hz}$ ).

3.3. Effect of Quinine on the Fast Ripples Observed in Animals with Spontaneous Seizures. The animals that received quinine exhibited vibrissae movements and they remained in a state of sleep for most of the analysis, although level 5 spontaneous seizures of the Racine scale were observed in some of these animals.

Like the animals that received CBX, spontaneous FRs were evident in all the regions analyzed when rats received quinine (Figure 2(b)), although there were fewer FRs during quinine administration in the RAH and $150-165$ min after quinine administration (Figures 3 and 4: during $4.8 \pm 1.4$ versus $1.0 \pm 0.3$; after $150-165 \min 4.6 \pm 1.7$ versus $1.7 \pm 0.4$ ). By contrast, there were fewer FRs in the RPH at all the EEG recording periods analyzed (during $5.0 \pm 1.5$ versus $1.0 \pm 0.5$; after $30-45 \min 6.0 \pm 1.6$ versus $3.0 \pm 0.7$; after $150-165 \mathrm{~min}$ $5.8 \pm 1.7$ versus $2.0 \pm 0.5$ ). While in the RPH a significant decrease in the mean number of oscillation cycles per FR was observed only during quinine administration ( $6.3 \pm 1.4$ versus $3.0 \pm 0.7)$ there was no difference in the mean frequency of FRs in the R AH and R PH. 


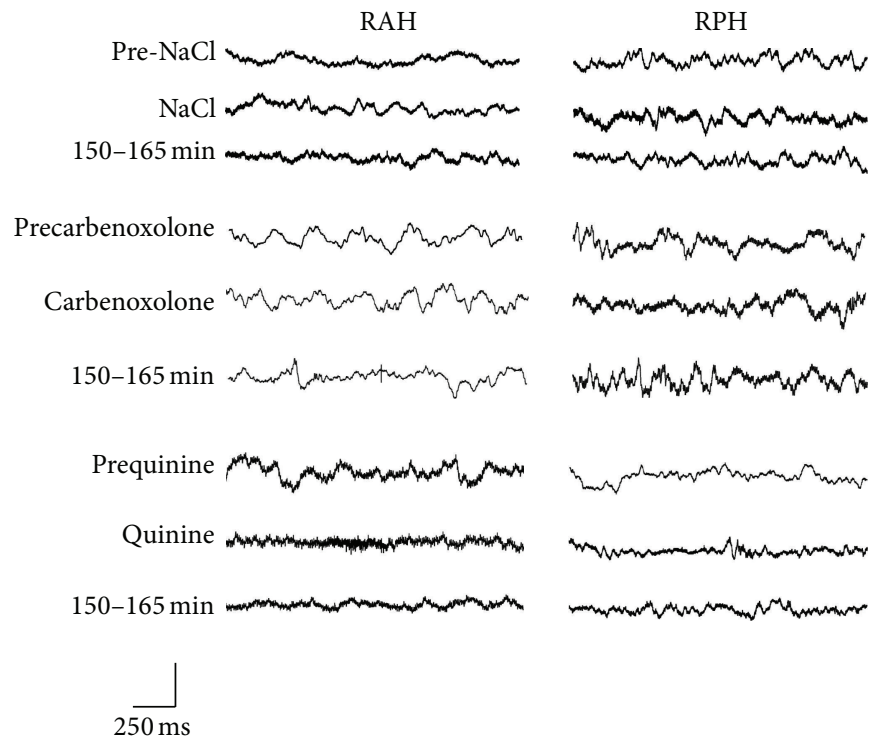

(a)
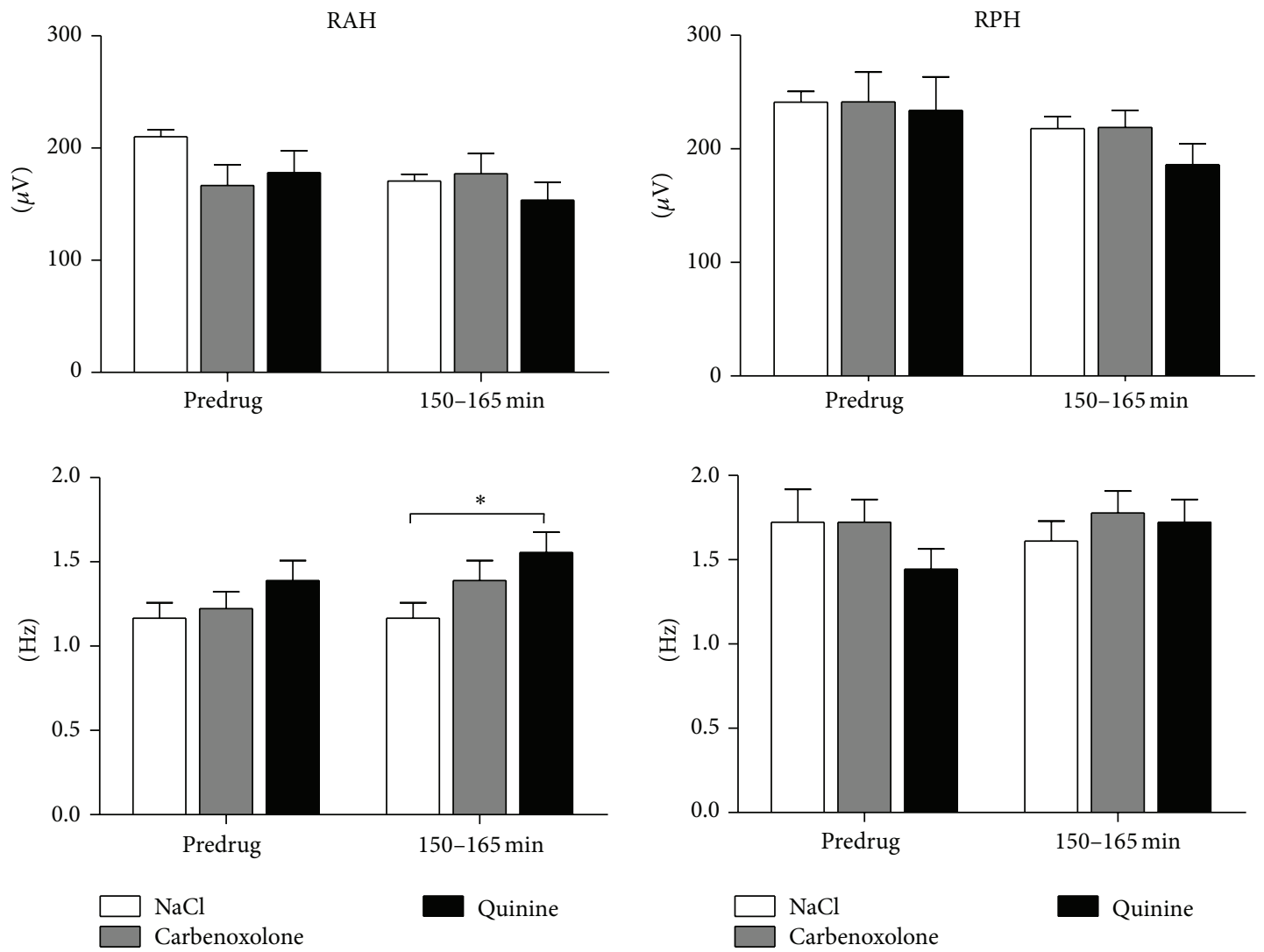

(b)

FIGURE 1: Representative intracranial EEG recordings from the different brain regions studied in three different rats from the NaCl, CBX, and quinine control groups ( $n=3$ each one). The lower graphs show the mean amplitude $(\mu \mathrm{V} \pm \mathrm{SEM})$ and frequency (Hz \pm SEM) of the electrical activity observed before (PRE-DRUG) and 150-165 min after drug administration. The $y$-axis calibration bar corresponds to the amplitude: $0.4 \mathrm{mV}$ for $\mathrm{NaCl}, 1 \mathrm{mV}$ for $\mathrm{CBX}$, and $0.5 \mathrm{mV}$ for quinine groups $\left({ }^{*} \mathrm{P}<0.05\right)$. 


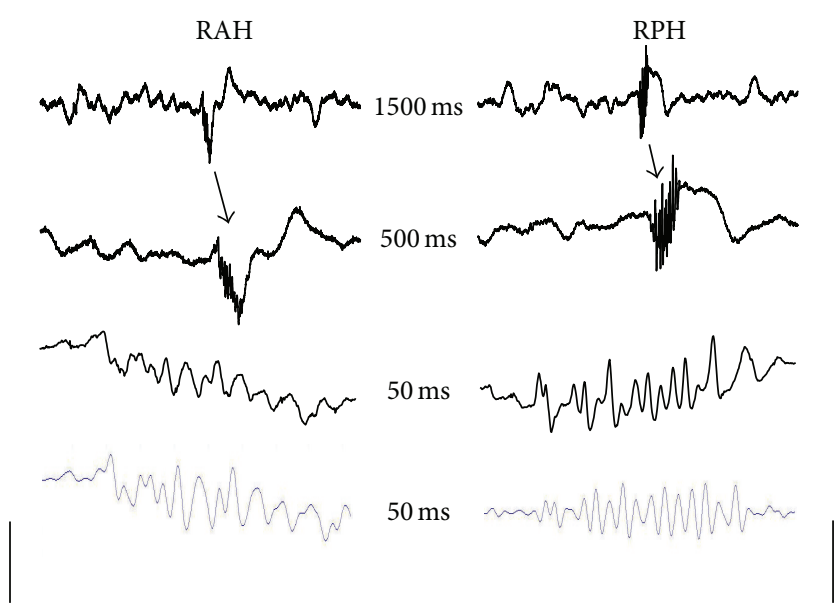

(a)

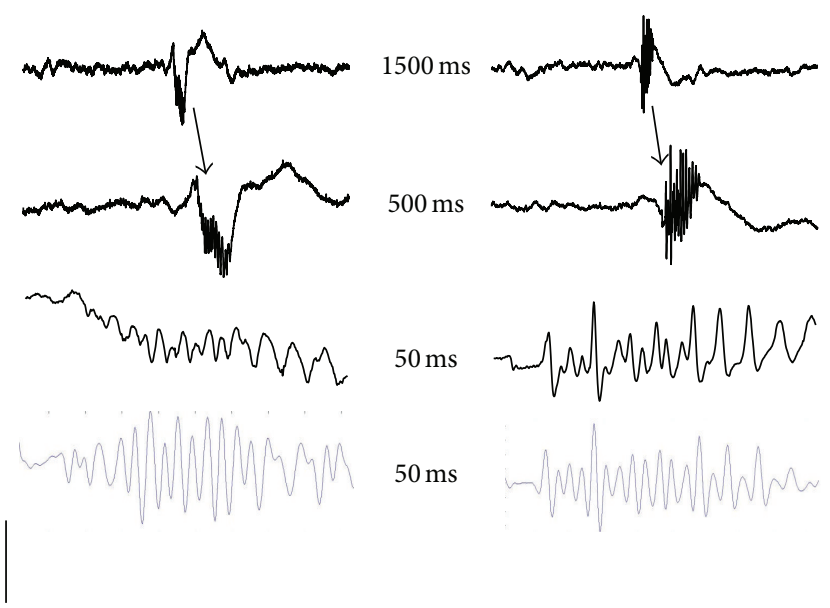

(b)

FIGURE 2: Representative intracranial EEG recordings taken before drug administration from the different brain regions studied in two rats from the experimental groups ( $n=6$ each one) with CBX (a) and quinine (b), in which spontaneous fast ripples (FRs) were observed simultaneously: right anterior hippocampus (RAH) and right posterior hippocampus (RPH). An EEG trace of $1.5 \mathrm{~s}$ with a spontaneous FR (arrow in the first trace) followed by extended EEG traces of the same activity. The last EEG trace is with an $80 \mathrm{~Hz}$ filter. The numbers in the centre correspond to the time of each EEG trace and the $y$-axis calibration bar corresponds to the amplitude: $1 \mathrm{mV}$ for the first and $0.4 \mathrm{mV}$ for the rest of the EEG traces.

\section{Discussion}

In the present study, no differences were found in the behavior observed in animals from control groups and electrical activity was characterized by the presence of slow physiological waves of low amplitude and frequency, although a slight increase in the frequency was observed between the saline solution control animals and those that received quinine. However, this difference could be due to different behavioral states of control animals considering that these animals were observed quiet and asleep, 150-165 min after drug administration. Moreover this difference did not appear to be physiologically relevant for our study given that the frequency of both groups did not exceed $2 \mathrm{~Hz}$.

In this study spontaneous FRs were detected in all the hippocampal brain regions analyzed from animals with spontaneous and recurrent seizures induced by the i.c.v. administration of pilocarpine, animals chosen after analyzing their intracranial EEG recordings with an algorithm exclusively designed to detect FRs. The movable recording microelectrodes that we used in the experimental animals were situated at different depths of hippocampal regions in order to detect FRs, which are generated in relatively small areas $\left(1 \mathrm{~mm}^{3}\right)$ $[15,16]$. The mean number and frequency of spontaneous FRs observed here were similar to those described previously in animals administered unilateral hippocampal injections of KA $[2,15]$. Moreover, unlike other studies, spontaneous FRs were observed during immobile, waking, and sleeping states of the animals, although periods of slow-wave sleep were not specifically analyzed, when FRs are more frequent $[15,38]$. There have been many studies into FRs and their probable mechanism of generation $[5,12-16,39]$, considering that FRs may be potential biomarkers for epilepsy and useful to detect candidate areas for resection [40]. Nevertheless, there are no in vivo studies about factors that could modulate FRs by electrical synapses.

We found that the nonspecific blocker of gap junctions, CBX, decreased the mean number of FRs in the RAH and $\mathrm{RPH}$, as well as the mean number of oscillation cycles per FR event, and the frequency in the RPH during and at different times after CBX administration. These data indicate a probable role of gap junctions in modulating FRs, although there is no data to date from studies in vivo with which the results of present study can be compared. Nevertheless, similar results were reported in some in vitro studies. In a study carried out on slices from epileptic patients, CBX $(0.2 \mathrm{mM})$ provoked a $50 \%$ decrease in FRs [31] as in other study [9], while ripple activity $(150-250 \mathrm{~Hz})$ was also blocked by the gap junction blocker halothane [41, 42]. Likewise, quinine decreased the mean number of FRs in the RAH and $\mathrm{RPH}$ during and at different times after its administration, while the mean number of oscillation cycles per FR event only decreased in the RPH, suggesting only a partial modulatory effect of quinine on FRs. This effect could be due to quinine blocking gap junctions formed only by $\mathrm{Cx} 36$, which are found in neurons, and we speculate that gap junctions formed by different connexins are required for FR activity, as observed here through the effects of the nonspecific blocker, CBX.

There are currently no in vivo studies to compare our results regarding the effects of quinine, although there is significant data supporting the important role of gap junctions in epileptiform activity in different in vitro and in vivo models. Indeed, the disruption of electrical coupling induced 

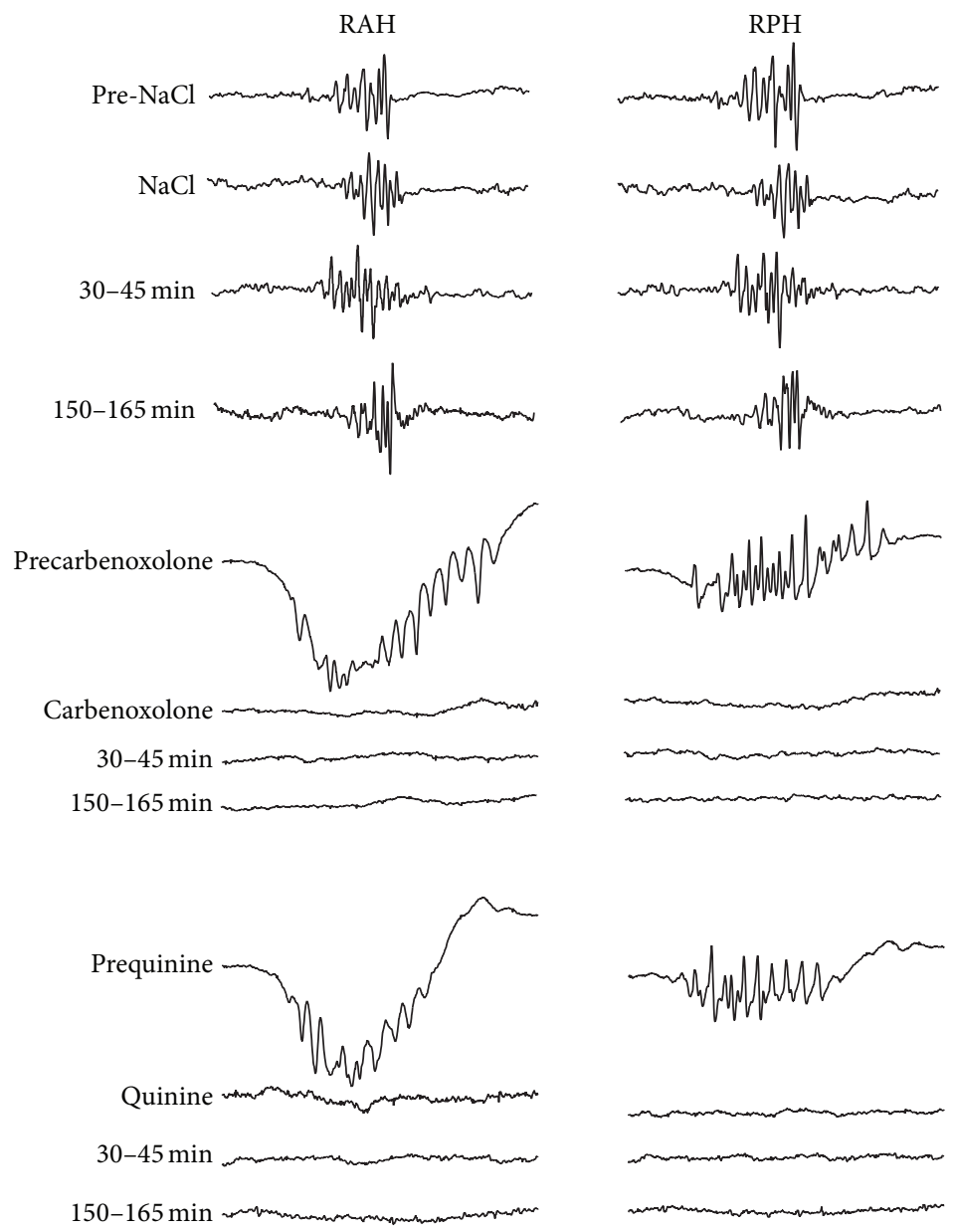

$\overline{25 s}_{\substack{\vdots \\ 8}}^{\substack{\Sigma \\ 8}}$

FIGURE 3: Representative intracranial EEG recordings from the different brain regions studied and obtained from three different rats in the $\mathrm{NaCl}, \mathrm{CBX}$, and quinine experimental groups ( $n=6$ each one) in which spontaneous fast ripples (FRs) were observed: right anterior hippocampus (RAH) and right posterior hippocampus (RPH) before, during, and at different times after drug administration. Note the effects of CBX and quinine on the FRs.

by gap junction blockers or through a deficiency in certain $\mathrm{Cx}$ proteins that make up gap junctions has antiepileptic effects [20, 24, 27-30]. Similarly, a role for Cx36 was shown in the generation of high frequency oscillations $(100-200 \mathrm{~Hz})$ and epileptiform field bursts recorded in slices from the hippocampus of deficient mice [26]. other in silico study has demonstrated the importance of axoaxonal gap junctions in FR generation [31]. These data support our data regarding the modulation of FRs by gap junctions, although we cannot rule out the influence of other factors that could modulate FRs, such as the chemical neurotransmission. In relation to this, serotonin was found to reduce electric coupling through protein kinase $\mathrm{C}$, via $\mathrm{IP}_{3} / \mathrm{Ca}^{2+}[43]$, and probable through 5-HT2 receptor activation [44]. In addition, the frequency of FRs was higher during slow wave sleep, a period in which serotonin levels are low in the model of chronic seizures induced by KA [2, 11, 15]. Indeed, a modulatory effect of serotonin was observed on electric synapses in weakly electrical coupled neural networks of the Helisoma ganglia [45]. In conjunction with our data regarding serotonin and FR modulation [46], these facts also suggest a possible influence of serotonin neurotransmission on gap junction activity and, hence, in the modulation of FRs.

The CBX and quinine have been clinically used against malaria and ulcers but they have not been completely tested for their anticonvulsant effects in patients; therefore it is necessary to carry out more in vivo experiments to find the role of gap junctions in the FRs modulation in order to consider this strategy as a possible clinical implication. 

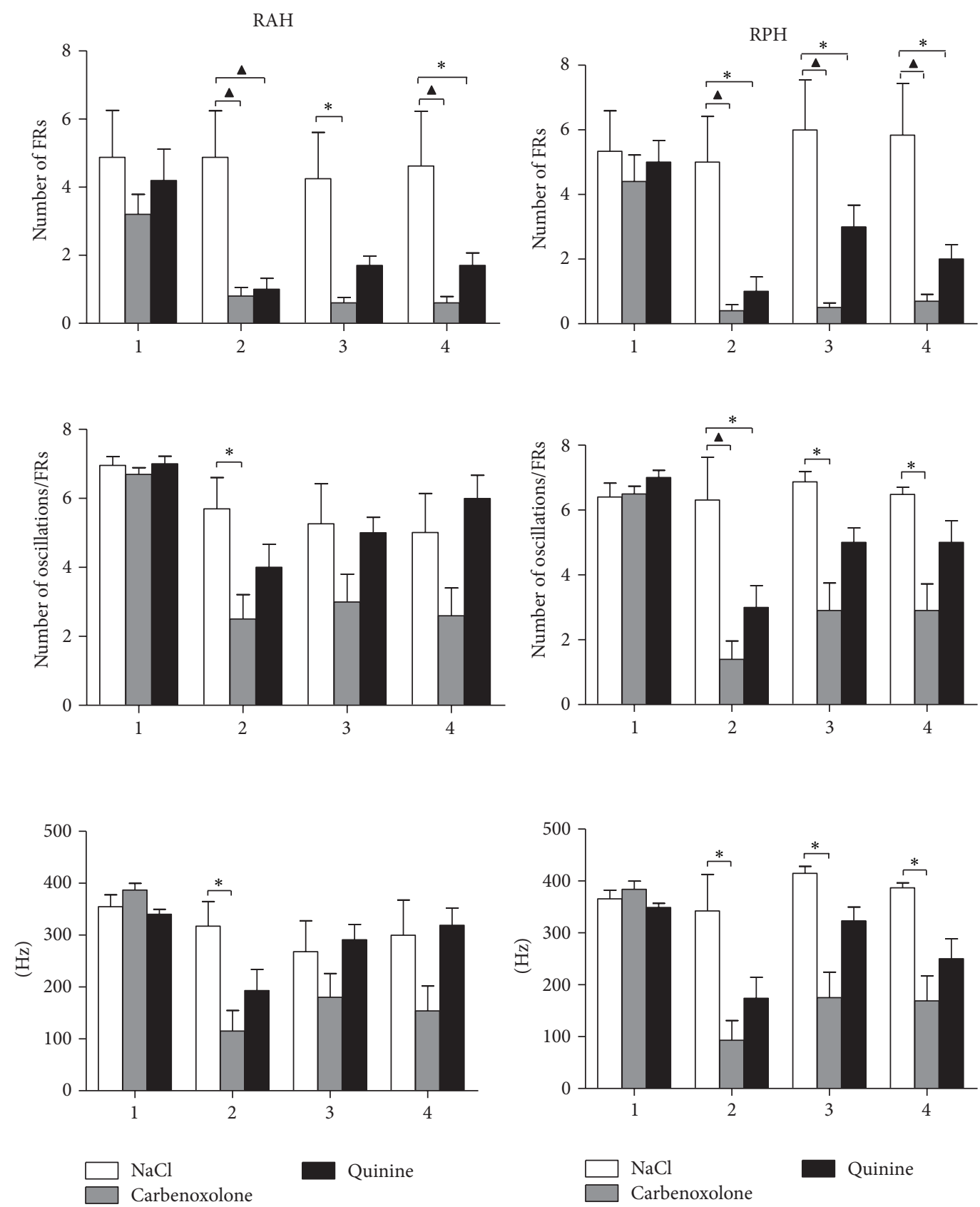

FIGURE 4: Graphs show the mean number of spontaneous FR events (spontaneous FRs \pm SEM) and the oscillation cycles per spontaneous $\mathrm{FR}$, as well as the mean frequency ( $\mathrm{Hz})$, before (1), during (2), and at different times after $\mathrm{NaCl}(\mathrm{NaCl}, 0.9 \%), \mathrm{CBX}$ (50 nmoles), and quinine (35 pmoles) administration (3, 30-45 min; 4, 150-165 min) in the regions studied: right anterior hippocampus (RAH) and right posterior hippocampus (RPH). A significant decrease in FR number was provoked by $\mathrm{CBX}$ and quinine administration in the $\mathrm{RAH}$ and $\mathrm{RPH}\left({ }^{*} P<0.05\right.$; $\triangle P<0.001)$.

\section{Conclusions}

Through the present data, we conclude that gap junctions could exert a modulatory effect on FRs in the hippocampus of rats with spontaneous seizures induced by pilocarpine. While these effects probably occur through gap junctions formed by different connexins, we cannot rule out the possible participation of chemical synapses in FR modulation, such as that of serotonin neurotransmission. Finally, it is necessary to perform additional in vivo experiments to determine the precise role of gap junctions in the modulation of FRs associated with epilepsy in the hippocampus.

\section{Conflict of Interests}

The authors declare that there is no conflict of interests regarding the publication of this paper. 


\section{Authors' Contribution}

Laura Medina-Ceja participated in supervising experiments, analysis and discussion of results, paper preparation, and final revision; Consuelo Ventura-Mejía contributed in experiments and analysis of results. Consuelo Ventura-Mejía and Laura Medina-Ceja contributed equally to this work.

\section{Acknowledgments}

This study was supported by Grant sponsor CONACYT-SEPCB, Grant no. 106179 to Laura Medina-Ceja.

\section{References}

[1] A. Bragin, J. Engel Jr., and R. J. Staba, "High-frequency oscillations in epileptic brain," Current Opinion in Neurology, vol. 23, no. 2, pp. 151-156, 2010.

[2] A. Bragin, J. Engel Jr., C. L. Wilson, E. Vizentin, and G. W. Mathern, "Electrophysiologic analysis of a chronic seizure model after unilateral hippocampal KA injection," Epilepsia, vol. 40, no. 9, pp. 1210-1221, 1999.

[3] A. Bragin, C. L. Wilson, J. Almajano, I. Mody, and J. Engel Jr., "High-frequency oscillations after status epilepticus: epileptogenesis and seizure genesis," Epilepsia, vol. 45, no. 9, pp. 10171023, 2004.

[4] D. F. He, D. L. Ma, Y. C. Tang, J. Engel Jr., A. Bragin, and F. R. Tang, "Morpho-physiologic characteristics of dorsal subicular network in mice after pilocarpine-induced status epilepticus," Brain Pathology, vol. 20, no. 1, pp. 80-95, 2010.

[5] P. Jiruska, A. D. Powell, W.-C. Chang, and J. G. R. Jefferys, "Electrographic high-frequency activity and epilepsy," Epilepsy Research, vol. 89, no. 1, pp. 60-65, 2010.

[6] J. D. Frost Jr., C. L. Lee, R. A. Hrachovy, and J. W. Swann, "High frequency EEG activity associated with ictal events in an animal model of infantile spasms," Epilepsia, vol. 52, no. 1, pp. 53-62, 2011.

[7] J. D. Frost, C. L. Lee, J. T. Le, R. A. Hrachovy, and J. W. Swann, "Interictal high frequency oscillations in an animal model of infantile spasms," Neurobiology of Disease, vol. 46, no. 2, pp. 377-388, 2012.

[8] R. D. Traub and A. Bibbig, "A model of high-frequency ripples in the hippocampus based on synaptic coupling plus axonaxon gap junctions between pyramidal neurons," Journal of Neuroscience, vol. 20, no. 6, pp. 2086-2093, 2000.

[9] A. K. Roopun, J. D. Simonotto, M. L. Pierce et al., "A nonsynaptic mechanism underlying interictal discharges in human epileptic neocortex," Proceedings of the National Academy of Sciences of the United States of America, vol. 107, no. 1, pp. 338-343, 2010.

[10] A. Bragin, J. Engel Jr., C. L. Wilson, I. Fried, and G. Buzsáki, "High-frequency oscillations in human brain," Hippocampus, vol. 9, no. 2, pp. 137-142, 1999.

[11] A. Bragin, C. L. Wilson, R. J. Staba, M. Reddick, I. Fried, and J. Engel Jr., "Interictal high-frequency oscillations $(80-500 \mathrm{~Hz})$ in the human epileptic brain: entorhinal cortex," Annals of Neurology, vol. 52, no. 4, pp. 407-415, 2002.

[12] A. Bragin, P. Claeys, K. Vonck et al., "Analysis of initial slow waves (ISWs) at the seizure onset in patients with drug resistant temporal lobe epilepsy," Epilepsia, vol. 48, no. 10, pp. 1883-1894, 2007.
[13] A. Bragin, C. L. Wilson, and J. Engel Jr., "Voltage depth profiles of high-frequency oscillations after kainic acid-induced status epilepticus," Epilepsia, vol. 48, supplement 5, pp. 35-40, 2007.

[14] D. E. Bragin, R. C. Bush, W. S. Müller, and E. M. Nemoto, "High intracranial pressure effects on cerebral cortical microvascular flow in rats," Journal of Neurotrauma, vol. 28, no. 5, pp. 775-785, 2011.

[15] A. Bragin, C. L. Wilson, and J. Engel Jr., "Chronic epileptogenesis requires development of a network of pathologically interconnected neuron clusters: a hypothesis," Epilepsia, vol. 41, no. 6, pp. S144-S152, 2000.

[16] A. Bragin, I. Mody, C. L. Wilson, and J. Engel Jr., "Local generation of fast ripples in epileptic brain," Journal of Neuroscience, vol. 22, no. 5, pp. 2012-2021, 2002.

[17] A. Bragin, C. L. Wilson, and J. Engel Jr., "Spatial stability over time of brain areas generating fast ripples in the epileptic rat," Epilepsia, vol. 44, no. 9, pp. 1233-1237, 2003.

[18] R. S. Fisher, W. R. S. Webber, R. P. Lesser, S. Arroyo, and S. Uematsu, "High-frequency EEG activity at the start of seizures," Journal of Clinical Neurophysiology, vol. 9, no. 3, pp. 441-448, 1992.

[19] R. D. Traub, A. Bibbig, A. Piechotta, A. Draguhn, and D. Schmitz, "Synaptic and nonsynaptic contributions to giant IPSPs and ectopic spikes induced by 4 -aminopyridine in the hippocampus in vitro," Journal of Neurophysiology, vol. 85, no. 3, pp. 1246-1256, 2001.

[20] J. L. Perez Velazquez and P. L. Carlen, "Gap junctions, synchrony and seizures," Trends in Neurosciences, vol. 23, no. 2, pp. 68-74, 2000.

[21] G. R. Juszczak and A. H. Swiergiel, "Properties of gap junction blockers and their behavioural, cognitive and electrophysiological effects: animal and human studies," Progress in NeuroPsychopharmacology and Biological Psychiatry, vol. 33, no. 2, pp. 181-198, 2009.

[22] M. Srinivas, M. G. Hopperstad, and D. C. Spray, "Quinine blocks specific gap junction channel subtypes," Proceedings of the National Academy of Sciences of the United States of America, vol. 98, no. 19, pp. 10942-10947, 2001.

[23] F. M. Ross, P. Gwyn, D. Spanswick, and S. N. Davies, "Carbenoxolone depresses spontaneous epileptiform activity in the CA1 region of rat hippocampal slices," Neuroscience, vol. 100, no. 4, pp. 789-796, 2000.

[24] R. Köhling, S. J. Gladwell, E. Bracci, M. Vreugdenhil, and J. G. R. Jefferys, "Prolonged epileptiform bursting induced by $0-\mathrm{Mg} 2+$ in rat hippocampal slices depends on gap junctional coupling," Neuroscience, vol. 105, no. 3, pp. 579-587, 2001.

[25] D. G. Margineanu and H. Klitgaard, "Can gap-junction blockade preferentially inhibit neuronal hypersynchrony vs. excitability?" Neuropharmacology, vol. 41, no. 3, pp. 377-383, 2001.

[26] N. Maier, M. Güldenagel, G. Söhl, H. Siegmund, K. Willecke, and A. Draguhn, "Reduction of high-frequency network oscillations (ripples) and pathological network discharges in hippocampal slices from connexin 36-deficient mice," Journal of Physiology, vol. 541, part 2, pp. 521-528, 2002.

[27] I. Pais, G. H. Sheriar, H. Monyer et al., "Sharp wave-like activity in the hippocampus in vitro in mice lacking the gap junction protein connexin 36," Journal of Neurophysiology, vol. 89, no. 4, pp. 2046-2054, 2003.

[28] Z. Gajda, E. Gyengési, E. Hermesz, K. Said Ali, and M. Szente, "Involvement of gap junctions in the manifestations and control 
of the duration of seizures in rats in vivo," Epilepsia, vol. 44, no. 12, pp. 1596-1600, 2003.

[29] L. Medina-Ceja, A. Cordero-Romero, and A. MoralesVillagrán, "Antiepileptic effect of carbenoxolone on seizures induced by 4 -aminopyridine: a study in the rat hippocampus and entorhinal cortex," Brain Research, vol. 1187, no. 1, pp. 74-81, 2008.

[30] L. Medina-Ceja and C. Ventura-Mejía, "Differential effects of trimethylamine and quinine on seizures induced by 4aminopyridine administration in the entorhinal cortex of vigilant rats," Seizure, vol. 19, no. 8, pp. 507-513, 2010.

[31] A. Simon, R. D. Traub, N. Vladimirov et al., "Gap junction networks can generate both ripple-like and fast ripple-like oscillations," European Journal of Neuroscience, vol. 39, no. 1, pp. 46-60, 2014.

[32] L. Medina-Ceja, K. Pardo-Peña, and C. Ventura-Mejía, "Evaluation of behavioral parameters and mortality in a model of temporal lobe epilepsy induced by intracerebroventricular pilocarpine administration," NeuroReport, 2014.

[33] R. J. Racine, "Modification of seizure activity by electrical stimulation: II. Motor seizure," Electroencephalography and Clinical Neurophysiology, vol. 32, no. 3, pp. 281-294, 1972.

[34] J. Csicsvari, H. Hirase, A. Czurkó, A. Mamiya, and G. Buzsáki, "Oscillatory coupling of hippocampal pyramidal cells and interneurons in the behaving rat," The Journal of Neuroscience, vol. 19, no. 1, pp. 274-287, 1999.

[35] R. J. Staba, C. L. Wilson, A. Bragin, I. Fried, and J. Engel Jr., "Quantitative analysis of high-frequency oscillations $(80-500 \mathrm{~Hz})$ recorded in human epileptic hippocampus and entorhinal cortex," Journal of Neurophysiology, vol. 88, no. 4, pp. 1743-1752, 2002.

[36] A. López-Cuevas, B. Castillo-Toledo, L. Medina-Ceja, C. Ventura-Mejía, and K. Pardo-Peña, "An algorithm for on-line detection of high frequency oscillations related to epilepsy," Computer Methods and Programs in Biomedicine, vol. 110, no. 3, pp. 354-360, 2013.

[37] S. M. Pincus, "Approximate entropy as a measure of system complexity," Proceedings of the National Academy of Sciences of the United States of America, vol. 88, no. 6, pp. 2297-2301, 1991.

[38] M. Lévesque, A. Bortel, J. Gotman, and M. Avoli, "Highfrequency $(80-500 \mathrm{~Hz})$ oscillations and epileptogenesis in temporal lobe epilepsy," Neurobiology of Disease, vol. 42, no. 3, pp. 231-241, 2011.

[39] G. Foffani, Y. G. Uzcategui, B. Gal, and L. Menendez de la Prida, "Reduced spike-timing reliability correlates with the emergence of fast ripples in the rat epileptic hippocampus," Neuron, vol. 55, no. 6, pp. 930-941, 2007.

[40] T. Akiyama, B. McCoy, C. Y. Go et al., "Focal resection of fast ripples on extraoperative intracranial EEG improves seizure outcome in pediatric epilepsy," Epilepsia, vol. 52, no. 10, pp. 1802-1811, 2011.

[41] A. Draguhn, R. D. Traub, D. Schmitz, and J. G. R. Jefferys, "Electrical coupling underlies high-frequency oscillations in the hippocampus in vitro," Nature, vol. 394, no. 6689, pp. 189-192, 1998.

[42] A. Ylinen, A. Bragin, Z. Nádasdy et al., "Sharp wave-associated high-frequency oscillation $(200 \mathrm{~Hz})$ in the intact hippocampus: network and intracellular mechanisms," The Journal of Neuroscience, vol. 15, part 1, pp. 30-46, 1995.

[43] B. Rörig and B. Sutor, "Serotonin regulates gap junction coupling in the developing rat somatosensory cortex," European Journal of Neuroscience, vol. 8, no. 8, pp. 1685-1695, 1996.
[44] B. Rörig and B. Sutor, "Nitric oxide-stimulated increase in intracellular cGMP modulates gap junction coupling in rat neocortex," NeuroReport, vol. 7, no. 2, pp. 569-572, 1996.

[45] T. M. Szabo, J. S. Caplan, and M. J. Zoran, "Serotonin regulates electrical coupling via modulation of extrajunctional conductance: H-current," Brain Research, vol. 1349, pp. 21-31, 2010.

[46] K. Pardo-Peña, L. Medina-Ceja, and A. Morales-Villagrán, "Serotonin modulates fast ripple activity in rats with spontaneous recurrent seizures," Brain Research, 2014. 

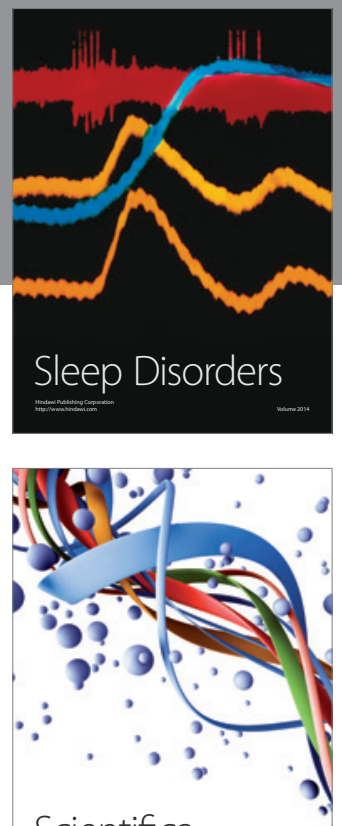

Scientifica
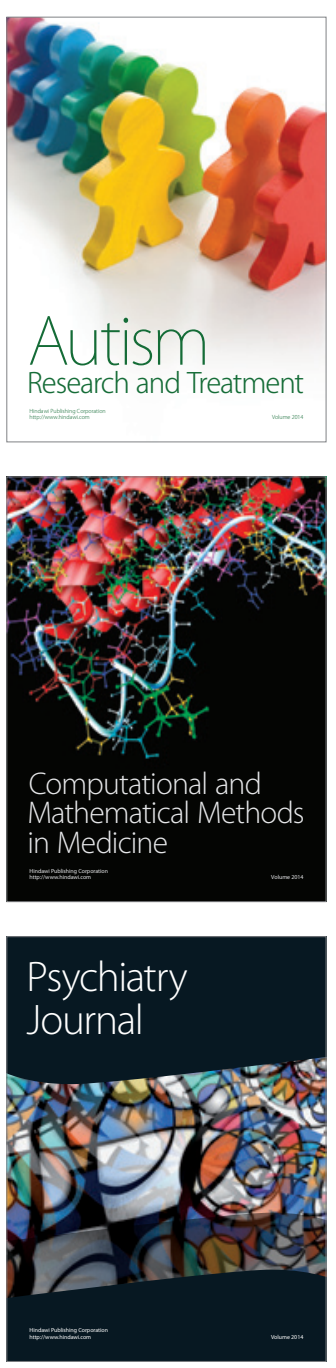
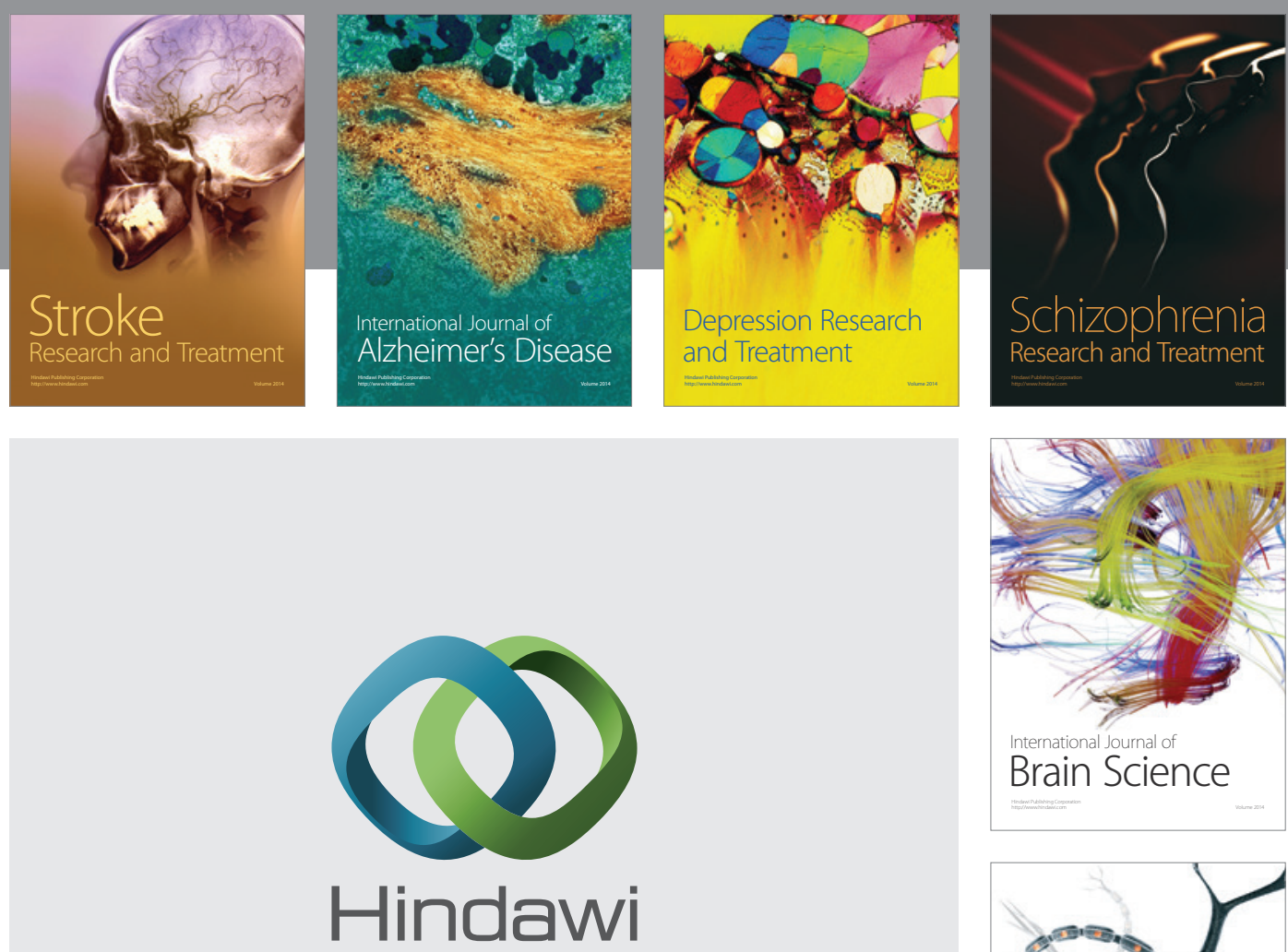

Submit your manuscripts at

http://www.hindawi.com
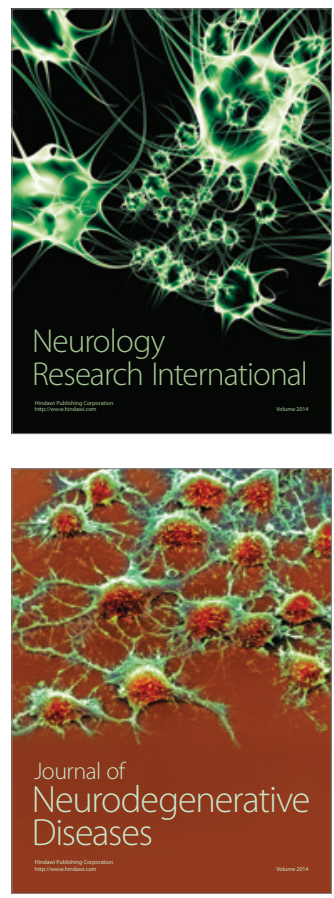

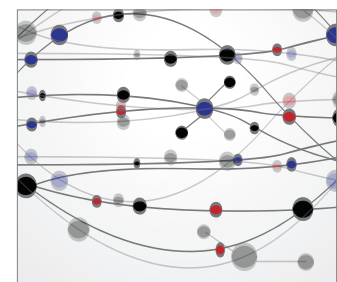

The Scientific World Journal
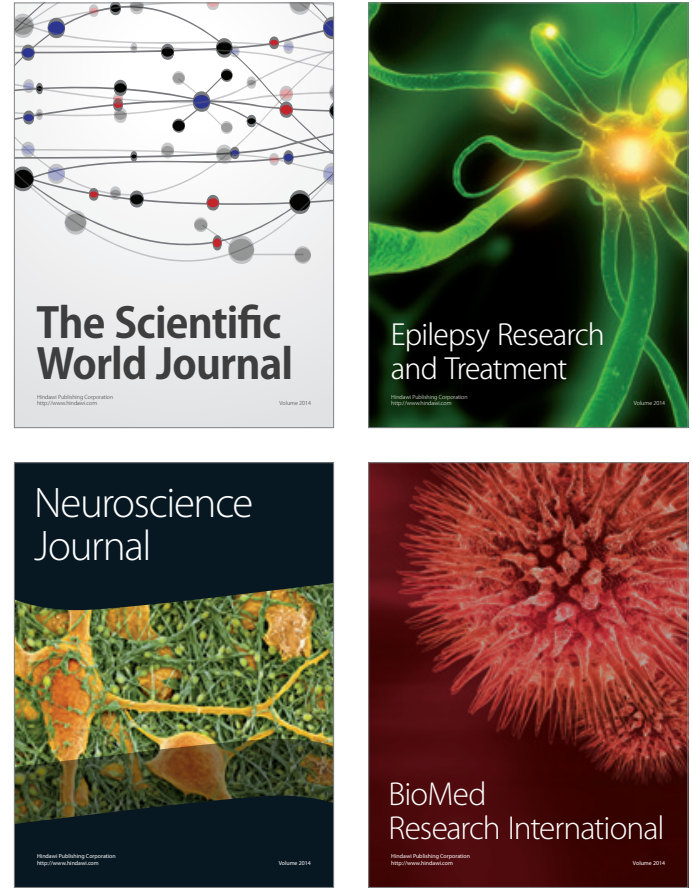

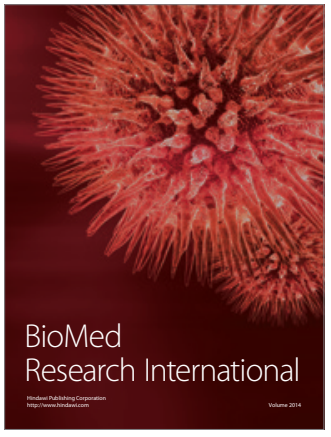

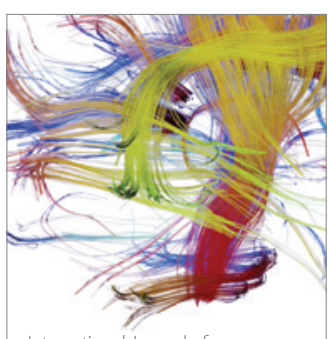

Brain Science

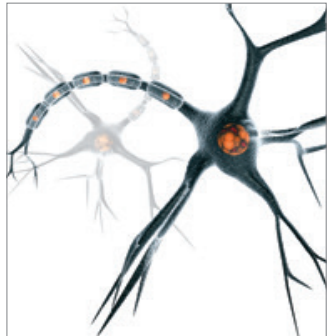

Neural Plasticity
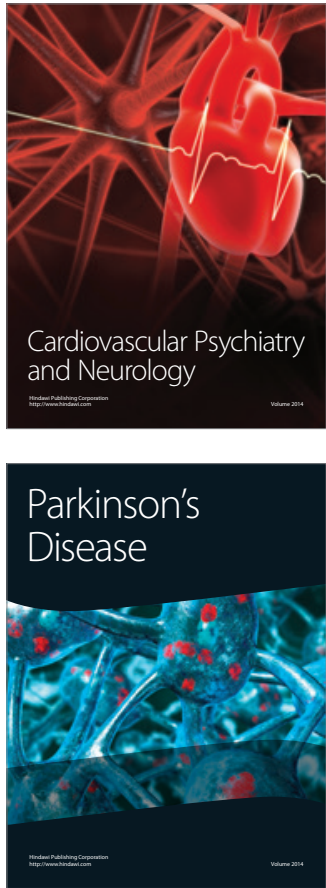